\title{
THE ROLE OF PATERNALISTIC LEADERSHIP IN EMPLOYEE VOICE AND SILENCE MOTIVES: DOES SURFACE SIMILARITY MATTERS?
}

\author{
Saadia Babur ${ }^{1 *}$, Zainab Bibi ${ }^{2}$, Jahanvash Karim ${ }^{3}$ \\ ${ }^{1 *}$ Ph.D., Scholar, Institute of Management Sciences, University of Balochistan, Quetta, Pakistan, and Assistant \\ Professor BUITEMS, Pakistan; ${ }^{2}$ Professor, Institute of Management Sciences, University of Balochistan, Quetta, \\ Pakistan; ${ }^{3}$ Professor, Institute of Management Sciences, University of Balochistan, Quetta, Pakistan. \\ Email: ${ }^{1 *}$ sadia.buitems@gmail.com, ${ }^{2}$ zainab.ims@uob.edu.pk, ${ }^{3}$ j_vash@ @otmail.com
}

Article History: Received on $16^{\text {th }}$ June 2021, Revised on $29^{\text {th }}$ June 2021, Published on $30^{\text {th }}$ June 2021

\begin{abstract}
Purpose of the study: This study compared the effect of paternalistic leadership on employee voice and silence motives based upon gender-based similarity attraction account among employee-leader dyads in Higher Educational Institutions (HEI). The study was conducted in public sector universities of Balochistan, Pakistan.

Methodology: The Study used Partial least squares-structural equation modelling, along with advanced methods for multi-group analysis, to assess and compare the proposed relationships between the gender similar and dissimilar dyads.

Main findings: The results of this study revealed significant differences between groups for the effect of the authoritative dimension of paternalism on pro-social silence, benevolence dimension of paternalism on quiescence silence and quiescence voice, and morality dimension on opportunistic silence.
\end{abstract}

Application of the study: The current study might help HEI authorities in understanding the effects of paternalistic leadership and diversity management.

Novelty/Originality of the study: This study makes a significant theoretical contribution by comparing the effect of paternalistic leadership on voicing motives of employees based on similarity attraction account between gender similar and dissimilar leader-subordinate dyads.

Keywords: Paternalistic Leadership, Employee Voice Motives, Employee Silence Motives, Multigroup Analysis (MGA), Partial Least Squares, Structural Equation Modelling (PLS-SEM).

\section{INTRODUCTION}

The role of Higher Educational Institutions (HEI) in the development of the country is vital. But these institutions need to be managed differently from business organizations. For this, the leadership of HEIs needs to be developed in a way that can facilitate faculty to meet their targets effectively rather than traditionally controlling them. Although, leadership has been researched from a long time, but most of the researchers are using western developed leadership theories in the eastern context without considering differences among norms embedded in individualistic and collectivistic cultures (Zhang, Tsui \& Wang, 2011). So, there is a need to investigate culturally embedded theories to develop more relevant policies. Therefore, the study intended to fill this gap by investigating the influence of paternalistic leadership style on employee intention to speak out that is embedded in cultural norms of the Pakistani context (Aycan, Kanungo, Mendonca, Yu, Deller, Stahl \& Kurshid, 2000). The research revealed that employees' voices have a diverse relationship with power concentration or reconcentration in the institutional procedure and process including allocation of resources and determining input related with the main strategies and practices. In this connection, the organizational conducive environment for promoting employees' voice can be dependent upon the leadership wherein paternalistic leadership is widely explored as more effective which have been widely recommended in previous literature suggests that leadership is the main antecedents of employee voice.

Does the question arise that in organizations whether the employees have the right to claim the share in decision-making that might mark the organizational activities under the dynamic leadership? The employees normally face circumstances wherein they decide to express their views or stay silent about work-related important issues (Chen, \& Hou 2016). Here, the role of leadership becomes important in considering the views of the employees about the important issues related with the needs at working place which in turn helps in augmenting their behaviour leading to the higher performances (Liu, Zhu \& Yang, 2010). In the higher educational context, the employees' voices are considered as a leading issue that remained the focus of interest for researchers during the past decades that denotes the employees' participation in decision-making which may ultimately influence group innovation, job attitudes as well as organizational development. In this regard, the role of leadership has been widely recognized as an influential phenomenon in response to the employees' voices and silence motives. However, there is a dire need to examine that among many leadership styles (Detert \& Burris, 2007), which leader trait is more effective whether ethical leadership (Chen \& Hou, 2016) or paternalistic leadership style (Chen, 2017). Thus, this study aimed to examine the role of paternalistic leadership style in determining the employees voice and silence motives in the higher education institutional context. 


\section{Research Objectives}

1. To examine the role of paternalistic leadership in determining the employees voice in the higher educational institutional context.

2. To examine the role of paternalistic leadership in determining the silence motives in the higher educational institutional context.

3. To what extent, does the surface similarity matter in connection to paternalistic leadership in employee voice and silence motives.

\section{LITERATURE REVIEW}

\section{Paternalistic Leadership Style}

Pakistan is a collectivist society with a high power distance orientation where people are inclined towards the ingroup collectivism (Aycan et al., 2000; Gorodnichenko \& Roland, 2012; Lyon, 2002). These values are considered as core values of paternalistic culture (Aycan et al., 2000) and are widely prevailed in Pakistani culture (Lyon, 2002). Farh and Cheng (2000) define paternalistic leadership as a "style that combines strong discipline and authority with fatherly benevolence and moral integrity" (Farh \& Cheng, 2000, p. 94). Gelfand et al., (2007) define paternalism as a "hierarchical relationship in which a leader guides professional and personal lives of subordinates in a manner resembling a parent, and in exchange expects loyalty and deference" (p. 493). The leadership styles have a significant impact on the attitude and behaviour of the employees in the organizational context wherein some styles are effective while some are ineffective based upon the situation and context. In this connection, the paternalistic leader acts like a father in a traditional family setup who is responsible to guide, protect and improve the lives of employees even in personal spheres (Farh \& Cheng, 2000; Kerfoot \& Knights, 1993).

\section{Employee Voice Behavior}

Voice behaviour refers to the arbitrary transmission of information that is intended to improve the organizational function to someone within the organization, who is responsible to take action (Detert \& Burris, 2007). In some situations, this voice behaviour may challenge and upset the status quo of the organization and power holders (Detert \& Burris, 2007). But this extra-role behaviour is vital to generate a diversity of opinions for making high-quality decisions (Morrison \& Milliken 2000). Normally voice behaviour is perceived to be a challenge-oriented behaviour, as the majority of the time, it might contain some suggestions for the improvements, criticism, and questions. Due to this, employees often hesitate to discuss their observations and opinions about organizational matters (Milliken et al., 2003; Morrison and Milliken, 2000). Even before raising voice, they carefully evaluate perceived resultant costs and benefits associated with voice (Dutton, Ashford, and Lawrenge 2001; Milliken, Morrison, and Hewlin 2003; Morrison and Milliken 2000). They opt for silence if the expected cost in terms of negative outcomes devastates associated benefits (Dutton et al., 2001). In addition to this cost-benefit analysis, employees may also have some hidden motives for making decisions to raise or hold a voice. Dyne et al., (2003) classify various motives behind this decision along with passive and active behaviour of the employee. The acquiescent voice appears when employees are disengaged from the situation and even don't think to change it, quiescent/defensive voice refers to employee protective behaviour adopted by employee against external threats, while employee acts under pro-social motives when he/she raises concern for the benefits of organization or for other colleagues rather than for themselves (Wang et al., 2012).

\section{Employee Silence Behavior}

Employees' silence is referred to conditions where employees intentionally hide their knowledge, thoughts, and concerns about organizational affairs (Morrison \& Milliken, 2000). Pinder and Harlos (2001, p. 334) have defined silence as "the withholding of any form of genuine expression about the individual's behavioural, cognitive and/or affective evaluations of his or her organizational circumstances to persons who are perceived to be capable of effecting change or redress". Dyne et al., (2003) differentiate the concept of employee silence from the absence of voice. According to them, employee silence is an employee's intentional decision to withhold his complaints, suggestions, or opinion about work and work-related context. Tangirala and Ramanujam (2008) also agree with Dyne et al., (2003) and consider silence as deliberate withholding of ostensibly important information, questions, opinions, and suggestions by employees on their working spheres. Silence has many forms based upon employee's personal motives to withhold their voice (Dyne et al., 2003; Milliken et al., 2003; Pinder \& Harlos, 2001). While most of the previous researchers have considered a perceived risk as to the prime antecedent of silence behaviour (Brinsfield, 2013). The current research literature highlights other motives beyond the expected risk that may cause an employee to remain silent (He et al., 2019; Pinder \& Harlos, 2001).

Pinder and Harlos (2001)_differentiated silence along with the two motives including quiescence and acquiescence. Quiescence silence refers to an employee's response due to fear, while in acquiescence silence, the employee deeply attaches to the current position and does not even think about other alternatives (Pinder \& Harlos, 2001). Dyne et al., (2003) add the pro-social dimension of silence motive where employees opt for silence in favor of others rather than for personal reasons (Wang et al., 2012). Scholars have described, recently, some other forms of silence behaviours that focused to achieve desired personal goals (Kurzon, 1995). According to Knoll and van Dick (2013) employees may 
withhold information to sustain knowledge advantage or to avoid any resultant workload. Knoll and van Dick (2013) calls it opportunistic silence where an employee intends to achieve some hidden opportunistic objectives. This type of silence is also pervasive in academic culture (Campbell et al., 2002; Hernaus et al., 2019). So, this study followed the classification of silence by Knoll and van Dick (2013) by measuring silence motives along with acquiescent, quiescent, prosocial, and opportunistic silence.

\section{Demographic Match: Surface Similarity}

Individuals tend to categorize themselves and others based on some salient attributes such as demographic characteristics (Edwards et al., 2009; Tajfel \& Turner, 2004). This process of categorization results in a comparison between "us" versus "them" scenarios. People, based on these comparisons, form their preferences for social interactions (Abrams et al., 1990; Lau \& Murnighan, 2005). This theory provides researchers a ground to develop an understanding of human interactions and to predict their behaviours in social settings.

\section{CONCEPTUAL FRAMEWORK AND DEVELOPMENT OF HYPOTHESIS}

\section{Leader and Employee Voice/Silence Behavior}

Research literature highlights that leadership styles of leaders may have a direct influence over employee's response behaviour (Liu et al., 2010). Specially in organizations with traditional hierarchical systems, leaders have the power to plan and assign employee's job-related activities and even have influence over outcomes such as rewards or punishments (Chen and Hou 2016; Morrison, Wheeler-Smith, and Kamdar 2011). Resource dependency theory supports this claim (Emerson 1962; Pfeffer and Salancik 2003; Detert and Burris 2007). According to this theory leaders' control over resources and outcomes give them power. Such leaders consider unsolicited voices from their subordinates either in form of questions or suggestions for improvements as challenging the status quo (Detert \& Burris, 2007; Milliken et al., 2003). Due to this, employees often opt to remain silent even when they have something important to share.

Additionally, leaders dominating in the authoritative behaviour prefers to have absolute authority and the control over organizational affairs, and employee's behaviour, may negatively reward subordinates when they do not follow his or her rules (Chan et al., 2013). Such leaders perceive voice as a challenge-oriented behaviour (Maynes \& Podsakoff, 2014) and try to induce fear and create an oppressed environment (Wu et al., 2011). Such leaders follow the traditional paradigm and expect subordinates to perform tasks according to given instructions. This may undermine employees' self-efficacy to perform their jobs (Zhang et al., 2011). Further, it may increase the inclination of employees to withhold additional efforts in performing tasks or refuse to exhibit discretionary behaviours that would benefit the organization (Ferris et al., 2009). Therefore, it can be presumed that employees who encounter authoritarian leaders are less likely to speak up.

Thus, it provides the basis for the first hypothesis that:

Hypothesis 1: A paternalistic leader having a high score on the authoritarian dimension is positively associated with silence behaviour.

Furthermore, the benevolence dimension of paternalistic behaviour is related with protection; support, and care provided by paternalistic leaders toward subordinates (Aycan et al., 1999). Social exchange theory provides the justification for the direct influence of benevolent leadership on employee voice behaviour (Emerson 1976). According to this, it can be claimed that a leader's benevolent behaviour supports employees even in personal spheres by enhancing work-life balance and in return, the leader is expected to be reciprocated by the constructive voice from the employee side. Hence it can be proposed that:

Hypothesis 2: Paternalistic leaders having a high score on the benevolence dimension is positively associated with employee voice.

Furthermore, the moral dimension of a paternalistic leader demonstrates high ethical standards (Cheng et al., 2004). Morality in the leader is demonstrated by high self-discipline and unselfish behaviour (Chan, 2014). These leaders demonstrate this behaviour by raising concerns and taking action against unethical acts (van Gils et al., 2015). They serve as a protagonist for their subordinates (Chan, 2014). According to social learning theory (Brown et al., 2005), subordinates learn behaviour from their superiors and try to replicate them. So employees feel encouraged to follow these high moral standards and feel safe to share opinions and suggestions regarding their work, work-related processes, and organizational context (Chen \& Hou, 2016). Hence it can be proposed that:

Hypothesis 3: Moral leadership is positively related to employee voice.

\section{Demographic Match as A Moderator Between Paternalistic Leadership and Employee Voice/ Silence Behavior}

Individuals in a social context are attracted toward others based upon some attributes. Social identity theory provides an explanation of how this process occurs. According to this, individuals tend to categorize themselves and others based on attributions such as demographics (Tajfel \& Turner, 2004). This creates the categorization of individuals among "us" 
versus "them" groups. Further individuals get positive self-esteem from the positive status of the in-group and will likely favor their in-group members and consider them trustworthy (Abrams et al., 1990; Lau \& Murnighan, 2005).

Individual's inclination toward in-group members develops an environment of trust among in-group members (Whitener et al., 1998). Additionally Whitener et al., (1998). propose that perceived similarity among managers and subordinates may influence trustworthiness among them (Duffy \& Ferrier, 2003). This perceived similarity has an impact on communication behaviour and the integration of members within a group (Jackson et al., 1991; O'Reilly et al., 1989; Zenger \& Lawrence, 1989). Members of the team who share similar characteristics are expected to communicate more as compared to others (Zenger \& Lawrence, 1989). Further, this demographical similarity attraction account influences performance evaluation of subordinates, correspondence by the supervisor, and ambiguity levels regarding task assignments and quality of social exchanges at the workplace (Judge \& Ferris, 1993; Liden et al., 1993; Tsui \& O'Reilly, $\underline{1989)}$.

McAllister (1995) also found that leaders are perceived to be more benevolent when they share more similar characteristics with subordinates. So, based upon the above findings it can be proposed that individuals having high demographic similarity with the leader will feel more trust and favourability. Such subordinates feel less reluctance while communicating their concerns. So, based upon all of this it can be proposed that:

Hypothesis 4: A paternalistic leader having a high score on the authoritarian dimension will foster more silence in dyadic relations having gender dissimilarity as compared to those having gender similarity.

Hypothesis 5: Paternalistic leaders having a high score on benevolence dimension will be more positively associated with employee voice and negatively associated with employee silence for the dyadic relations having gender dissimilarity as compared to those having gender similarity.

Hypothesis 6: Paternalistic leaders having a high score on morality dimension will be more positively associated with employee voice and more negatively associated with employee silence for the dyadic relations having gender dissimilarity as compared to those having gender similarity.

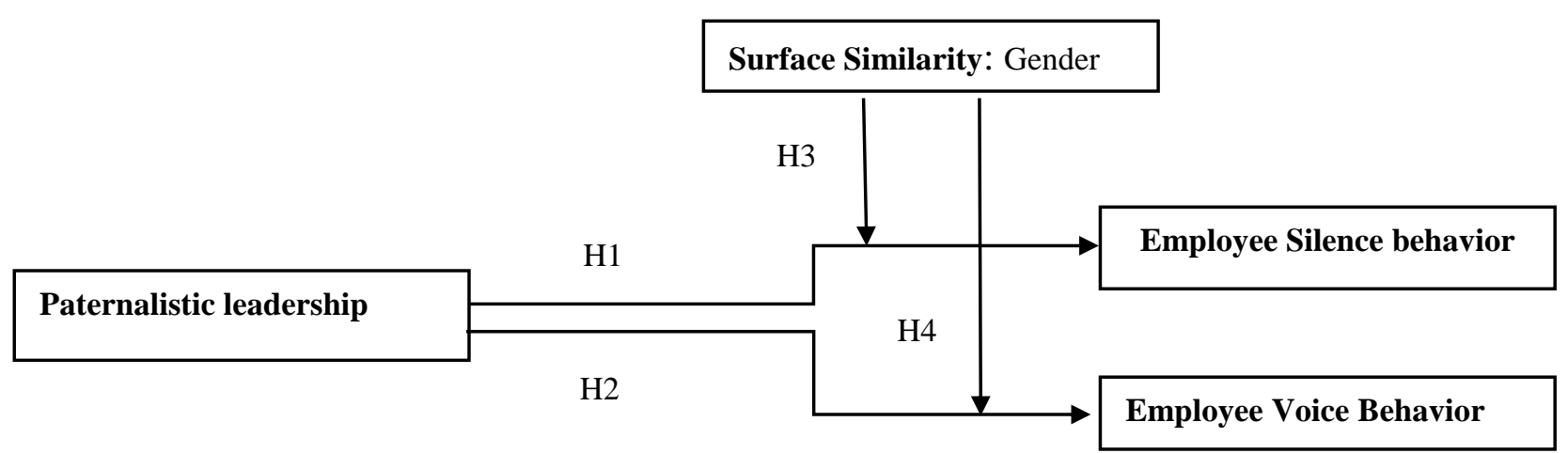

Figure 1: Hypothesized Model

There are 6 hypotheses above but the model is prepared for only 4 ?

\section{METHOD}

\section{Participants and Procedures}

The population of the study was university employees of Balochistan, Pakistan. Data was collected from the targeted sample by using the convenience sampling technique. For determining sample size recent research literature recommends conducting priori test for the expected power of the study (Chuan, 2006; Cohen, 1992; Prajapati et al., 2010). Conventional levels of effect size, alpha value, and power values provided by Cohen (1992) was used to determine the minimum sample size. Based upon these values minimum required sample size for this study was 119 . Although this sample size is enough to yield a power of .80 .

Along with this, Saunders et al., (2009) also highlight the possibility of non-response from target respondents. This may drop the number of usable questionnaires below the required numbers for analysis. To overcome this issue $\underline{S a u n d e r s,}$ Lewis, and Thornhill (2009) suggest raising in required sample size by adjusting the expected response rate. The response rate was estimated based upon response rates in surveys undertaken in Pakistani universities yielded for example $43.3 \%$ (Bano et al., 2017), 50\% (Karim et al., 2015), 64\%, (Bibi et al., 2013), 72\% (Arif \& Ilyas, 2013), 72\% (Afsar, 2014), 80\% (Farrukh et al., 2019). By taking an approximately average response rate, this study considered $61 \%$ estimated response rate. So total sample size of this study after consideration for the non-response rate was 196. 


\section{Measures}

\section{Paternalistic leadership style}

Perceived Paternalistic leadership behaviour of immediate supervisor was measured by adopting the 21 items Paternalistic Leadership Scale developed by Cheng et al., (2004) along with benevolence, moral and authoritarian dimensions. The results have been offered in table 1:

\section{Employee voice behavior}

Employee's voice behaviour was measured by using Dyne et al., (2003) scale consisting of 16 items to measures employee's voice behaviours. Wordings of the scale was slightly modified as this scale was originally designed to collect the supervisor's evaluation of employee's voice behaviour. The results have been provided in table 1 .

\section{Employee silence behavior}

Employee silence behaviour was measured by a scale developed by Knoll and van Dick (2013) that used 12 items to measure employee silence along with four dimensions. The results have been offered in table 1 .

\section{Demographic match}

Similarity-Attraction Account based on Gender: Avery et al., (2008) was followed to collect responses on gender, respondents were asked to mention their own and immediate supervisor's gender. The results have been provided in table 1 .

\section{Analysis Strategy}

The study used the Statistical Package for the Social Sciences (SPSS) for data management, and preliminary analysis. The main analysis was performed by following the structural equation modelling (SEM) technique by using SMART PLS software. SMART PLS is assumed to be a more preferred technique for complex models (Kline, 2011; Rigdon, 2016).

\section{RESULTS}

\section{Respondent Profile}

The results show that $65 \%$ of respondents were male and $35 \%$ were female. The respondents were categorized into five age groups: Less than 25 years (6.8\%), 25-34 years (50.3\%), 35-44 years (31.9\%), 45-54 years (7.6\%), and 55-64 years (3.4\%). Further, $10.7 \%$ of the respondents had completed their master's level degree, $49.7 \%$ MS/M.Phil. level education, 26.6\% had completed Ph.D. and $13 \%$ were having other qualifications. Moreover, 33.1\% of employees were having job experience of Less than 5 years, $28.2 \%$ were having an experience of 5 to 10 years, $22 \%$ of respondents had an experience of 10 to 15 years, $10.7 \%$ were having an experience of 15 to 20 years while $6.2 \%$ were having experience of more than 20 years.

\section{Model Assessment}

For assessing the models for gender similarity and dissimilarity dyads and comparison, the study used a three-step approach guided by Henseler et al., (2016) and Sarstedt et al., (2011).

\section{Measurement Model Assessment}

First, the acceptability of the measurement models for gender similarity and dissimilarity was confirmed (Hair et al. 2014). The measurement model used in this study included constructs: paternalistic leadership style, employee voice, and silence motives. First, factor loadings of each indicator on the respective latent variable (LV) were assessed. For acceptability, it is required to be higher than 0.7 (Hair, Ringle, and Sarstedt 2011). However, values below 0.4 should be considered for the removal of the item from the measurement model, while values in between 0.4 and 0.7 can be considered for removal from the model only if doing the same increases the CR and AVE above the threshold (Chin, 2010; Hair et al., 2011). Factor loadings for both measurement models are presented in Table 1.

Table 1: Assessment Results of the Measurement Model

\begin{tabular}{|c|c|c|c|c|c|c|c|c|c|c|}
\hline \multicolumn{11}{|c|}{ Assessment Results of the Measurement Model } \\
\hline $\begin{array}{l}\text { Construct/ } \\
\text { Associated } \\
\text { Items }\end{array}$ & Loading & & $\begin{array}{l}\text { Cronba } \\
\text { Alpha }\end{array}$ & & Rho_A & & CR & & AVE & \\
\hline $\begin{array}{l}\text { Paternalisti } \\
\text { c } \\
\text { Leadership }\end{array}$ & $\begin{array}{l}\text { Gender } \\
\text { Similar } \\
\text { ity }\end{array}$ & $\begin{array}{l}\text { GENDER } \\
\text { Dissimila } \\
\text { rity }\end{array}$ & $\begin{array}{l}\text { Gender } \\
\text { Similar } \\
\text { ity }\end{array}$ & $\begin{array}{l}\text { Gender } \\
\text { Dissimil } \\
\text { arity }\end{array}$ & $\begin{array}{l}\text { Gender } \\
\text { Similar } \\
\text { ity }\end{array}$ & $\begin{array}{l}\text { Gender } \\
\text { Dissimil } \\
\text { arity }\end{array}$ & $\begin{array}{l}\text { Gender } \\
\text { Similar } \\
\text { ity }\end{array}$ & $\begin{array}{l}\text { Gender } \\
\text { Dissimil } \\
\text { arity }\end{array}$ & $\begin{array}{l}\text { Gender } \\
\text { Similar } \\
\text { ity }\end{array}$ & $\begin{array}{l}\text { Gende } \\
\mathbf{r} \\
\text { Dissim } \\
\text { ilarity }\end{array}$ \\
\hline \multicolumn{11}{|c|}{ Benevolence } \\
\hline PLBN11 & 0.809 & 0.739 & 0.885 & 0.882 & 0.889 & 0.901 & 0.91 & 0.908 & 0.593 & 0.585 \\
\hline
\end{tabular}




\begin{tabular}{|c|c|c|c|c|c|c|c|c|c|c|}
\hline PLBN3 & 0.8 & 0.744 & & & & & & & & \\
\hline PLBN4 & 0.785 & 0.835 & & & & & & & & \\
\hline PLBN5 & 0.798 & 0.806 & & & & & & & & \\
\hline PLBN7 & 0.765 & 0.779 & & & & & & & & \\
\hline PLBN8 & 0.722 & 0.716 & & & & & & & & \\
\hline PLBN9 & 0.703 & 0.725 & & & & & & & & \\
\hline \multicolumn{11}{|c|}{ Authoritative } \\
\hline PLAU18 & 0.832 & 0.774 & \multirow[t]{4}{*}{0.807} & \multirow[t]{4}{*}{0.771} & \multirow[t]{4}{*}{0.828} & \multirow[t]{4}{*}{0.783} & \multirow[t]{4}{*}{0.873} & \multirow[t]{4}{*}{0.852} & \multirow[t]{4}{*}{0.632} & \multirow[t]{4}{*}{0.590} \\
\hline PLAU19 & 0.735 & 0.765 & & & & & & & & \\
\hline PLAU20 & 0.842 & 0.733 & & & & & & & & \\
\hline PLAU21 & 0.767 & 0.799 & & & & & & & & \\
\hline \multicolumn{11}{|l|}{ Moral } \\
\hline PLMR12 & 0.793 & 0.74 & \multirow[t]{3}{*}{0.688} & \multirow[t]{3}{*}{0.743} & \multirow[t]{3}{*}{0.699} & \multirow[t]{3}{*}{0.745} & \multirow[t]{3}{*}{0.828} & \multirow[t]{3}{*}{0.855} & \multirow[t]{3}{*}{0.617} & \multirow[t]{3}{*}{0.663} \\
\hline PLMR15 & 0.703 & 0.852 & & & & & & & & \\
\hline PLMR16 & 0.853 & 0.846 & & & & & & & & \\
\hline \multicolumn{11}{|c|}{ Acquiescence silence } \\
\hline SAQ1 & 0.824 & 0.824 & \multirow[t]{3}{*}{0.833} & \multirow[t]{3}{*}{0.807} & \multirow[t]{3}{*}{0.835} & \multirow[t]{3}{*}{0.821} & \multirow[t]{3}{*}{0.9} & 0.885 & 0.75 & 0.720 \\
\hline SAQ2 & 0.896 & 0.843 & & & & & & & & \\
\hline SAQ3 & 0.877 & 0.878 & & & & & & & & \\
\hline Opportun & ic silenc & & & & & & & & & \\
\hline SOP4 & 0.812 & 0.795 & 0.783 & 0.772 & 0.784 & 0.777 & 0.874 & 0.868 & 0.697 & 0.687 \\
\hline SOP5 & 0.85 & 0.842 & & & & & & & & \\
\hline SOP6 & 0.843 & 0.849 & & & & & & & & \\
\hline Pro-social & ence & & & & & & & & & \\
\hline SPR7 & 0.839 & 0.881 & 0.792 & 0.831 & 0.793 & 0.858 & 0.878 & 0.897 & 0.706 & 0.744 \\
\hline SPR8 & 0.848 & 0.869 & & & & & & & & \\
\hline SPR9 & 0.833 & 0.837 & & & & & & & & \\
\hline Quiescenc & ilence & & & & & & & & & \\
\hline SQ10 & 0.836 & 0.839 & 0.814 & 0.783 & 0.83 & 0.789 & 0.888 & 0.872 & 0.725 & 0.695 \\
\hline SQ11 & 0.858 & 0.839 & & & & & & & & \\
\hline SQ12 & 0.86 & 0.822 & & & & & & & & \\
\hline Acquiesce & Voice & & & & & & & & & \\
\hline VAQ2 & 0.816 & 0.841 & 0.749 & 0.745 & 0.762 & 0.746 & 0.855 & 0.854 & 0.663 & 0.662 \\
\hline VAQ4 & 0.791 & 0.795 & & & & & & & & \\
\hline VAQ5 & 0.836 & 0.802 & & & & & & & & \\
\hline Quiescenc & Toice & & & & & & & & & \\
\hline VQ6 & 0.748 & 0.776 & 0.614 & 0.623 & 0.649 & 0.631 & 0.787 & 0.798 & 0.554 & 0.568 \\
\hline VQ7 & 0.815 & 0.706 & & & & & & & & \\
\hline VQ9 & 0.663 & 0.778 & & & & & & & & \\
\hline
\end{tabular}

Further, the discriminate validity of constructs was assessed by using Fornell-Larcker criteria and Heterotrait-Monotrait (HTMT) ratio following the suggestions of Chin, 2010 and Hair et al., 2011. For the establishment of discriminate validity by following Fornell-Larcker criterion, the square root of the AVE of each construct must be greater than all the correlations among that construct and with other constructs in the model (Chin 2010; Hair et al. 2014) and HTMT ratio requires to be less than 0.90 (Henseler et al., 2015). Results of both methods reveal that models hold acceptable discriminant validity (results are presented in table 2 and table 3 ).

Table 2: Discriminant Validity (Fornell-Larcker Criterion)

\begin{tabular}{|c|c|c|c|c|c|c|c|c|c|c|c|c|c|c|c|c|c|c|}
\hline \multicolumn{19}{|c|}{ Discriminant Validity (Fornell-Larcker Criterion) } \\
\hline & \multicolumn{9}{|c|}{ Gender Similarity } & \multicolumn{9}{|c|}{ Gender Dissimilarity } \\
\hline $\begin{array}{l}\text { Con } \\
\text { stru } \\
\text { cts }\end{array}$ & $\begin{array}{l}\text { Acq } \\
\text { uiesc } \\
\text { ence } \\
\text { Silen } \\
\text { ce }\end{array}$ & $\begin{array}{l}\text { Acq } \\
\text { uies } \\
\text { cenc } \\
\text { e } \\
\text { Voic } \\
\text { e }\end{array}$ & $\begin{array}{l}\mathrm{Au} \\
\text { tho } \\
\text { rit } \\
\text { ari } \\
\text { an }\end{array}$ & $\begin{array}{l}\text { Be } \\
\text { ne } \\
\text { vo } \\
\text { len } \\
\text { ce }\end{array}$ & $\begin{array}{l}\mathrm{M} \\
\mathrm{o} \\
\mathrm{r} \\
\mathrm{a} \\
1\end{array}$ & $\begin{array}{l}\text { Opp } \\
\text { ortun } \\
\text { istic } \\
\text { Silen } \\
\text { ce }\end{array}$ & $\begin{array}{l}\text { Pro- } \\
\text { Soci } \\
\text { al } \\
\text { Sile } \\
\text { nce }\end{array}$ & $\begin{array}{l}\text { Qui } \\
\text { esce } \\
\text { nce } \\
\text { Sile } \\
\text { nce }\end{array}$ & $\begin{array}{l}\text { Qui } \\
\text { esce } \\
\text { nce } \\
\text { Voi } \\
\text { ce }\end{array}$ & $\begin{array}{l}\text { Acq } \\
\text { uiesc } \\
\text { ence } \\
\text { Silen } \\
\text { ce }\end{array}$ & $\begin{array}{l}\text { Acq } \\
\text { uies } \\
\text { cenc } \\
\mathrm{e} \\
\text { Voic } \\
\mathrm{e}\end{array}$ & $\begin{array}{l}\mathrm{Au} \\
\text { tho } \\
\text { rit } \\
\text { ari } \\
\text { an }\end{array}$ & $\begin{array}{l}\text { Be } \\
\text { ne } \\
\text { vo } \\
\text { len } \\
\text { ce }\end{array}$ & $\begin{array}{l}\text { M } \\
\text { or } \\
\text { al }\end{array}$ & $\begin{array}{l}\text { Opp } \\
\text { ortun } \\
\text { istic } \\
\text { Silen } \\
\text { ce }\end{array}$ & $\begin{array}{l}\text { Pro- } \\
\text { Soci } \\
\text { al } \\
\text { Sile } \\
\text { nce }\end{array}$ & $\begin{array}{l}\text { Qui } \\
\text { esce } \\
\text { nce } \\
\text { Sile } \\
\text { nce }\end{array}$ & $\begin{array}{l}\text { Qui } \\
\text { esce } \\
\text { nce } \\
\text { Voi } \\
\text { ce }\end{array}$ \\
\hline $\begin{array}{l}\text { Acq } \\
\text { uiesc } \\
\text { ence }\end{array}$ & & & & & & & & & & & & & & & & & & \\
\hline $\begin{array}{l}\text { Silen } \\
\text { ce }\end{array}$ & $\begin{array}{l}0.93 \\
1\end{array}$ & & & & & & & & & $\begin{array}{l}0.92 \\
1412\end{array}$ & & & & & & & & \\
\hline Acq & 0.34 & 0.90 & & & & & & & & 0.35 & 0.90 & & & & & & & \\
\hline
\end{tabular}




\begin{tabular}{|c|c|c|c|c|c|c|c|c|c|c|c|c|c|c|c|c|c|c|}
\hline $\begin{array}{l}\text { uiesc } \\
\text { ence } \\
\text { Voic } \\
\mathrm{e}\end{array}$ & 6 & 2 & & & & & & & & 2 & 1665 & & & & & & & \\
\hline $\begin{array}{l}\text { Auth } \\
\text { orita } \\
\text { rian }\end{array}$ & $\begin{array}{l}0.26 \\
4\end{array}$ & $\begin{array}{l}0.42 \\
5\end{array}$ & $\begin{array}{l}0.8 \\
91\end{array}$ & & & & & & & $\begin{array}{l}0.15 \\
2\end{array}$ & $\begin{array}{l}0.45 \\
9\end{array}$ & $\begin{array}{l}0.8 \\
76 \\
35 \\
6 \\
\end{array}$ & & & & & & \\
\hline $\begin{array}{l}\text { Bene } \\
\text { vole } \\
\text { nce }\end{array}$ & $\begin{array}{l}- \\
0.24 \\
8\end{array}$ & 0.15 & $\begin{array}{l}- \\
0.1 \\
12\end{array}$ & $\begin{array}{l}0.8 \\
77\end{array}$ & & & & & & $\begin{array}{l}- \\
0.41 \\
2\end{array}$ & $\begin{array}{l}- \\
0.00 \\
4\end{array}$ & $\begin{array}{l}0.0 \\
08\end{array}$ & $\begin{array}{l}0.8 \\
74 \\
64 \\
3\end{array}$ & & & & & \\
\hline $\begin{array}{l}\text { Mor } \\
\text { al }\end{array}$ & $\begin{array}{l}- \\
0.04 \\
7\end{array}$ & $\begin{array}{l}0.20 \\
7\end{array}$ & $\begin{array}{l}- \\
0.0 \\
5\end{array}$ & $\begin{array}{l}0.6 \\
16\end{array}$ & $\begin{array}{l}8 \\
8 \\
6\end{array}$ & & & & & -0.08 & $\begin{array}{l}0.08 \\
3\end{array}$ & $\begin{array}{l}- \\
0.1 \\
57\end{array}$ & $\begin{array}{l}0.4 \\
16\end{array}$ & $\begin{array}{l}0 . \\
9 \\
0 \\
2 \\
2 \\
1 \\
9\end{array}$ & & & & \\
\hline $\begin{array}{l}\text { Opp } \\
\text { ortun } \\
\text { istic } \\
\text { Silen } \\
\text { ce }\end{array}$ & $\begin{array}{l}0.24 \\
5\end{array}$ & $\begin{array}{l}0.02 \\
6\end{array}$ & $\begin{array}{l}0.0 \\
37\end{array}$ & $\begin{array}{l}- \\
0.7 \\
43\end{array}$ & $\begin{array}{l}5 \\
1 \\
4\end{array}$ & $\begin{array}{l}0.91 \\
3783\end{array}$ & & & & $\begin{array}{l}0.48 \\
5\end{array}$ & $\begin{array}{l}0.26 \\
8\end{array}$ & $\begin{array}{l}0.1 \\
49\end{array}$ & $\begin{array}{l}0.6 \\
39\end{array}$ & $\begin{array}{l}- \\
0 . \\
1 \\
8 \\
9\end{array}$ & $\begin{array}{l}0.91 \\
0494\end{array}$ & & & \\
\hline $\begin{array}{l}\text { Pro- } \\
\text { Soci } \\
\text { al } \\
\text { Silen } \\
\text { ce }\end{array}$ & $\begin{array}{l}0.36 \\
1\end{array}$ & $\begin{array}{l}0.47 \\
6\end{array}$ & $\begin{array}{l}0.5 \\
92\end{array}$ & $\begin{array}{l}- \\
0.0 \\
42\end{array}$ & $\begin{array}{l}0 \\
4 \\
2\end{array}$ & $\begin{array}{l}0.06 \\
5\end{array}$ & $\begin{array}{l}0.91 \\
651 \\
5\end{array}$ & & & $\begin{array}{l}0.22 \\
3\end{array}$ & $\begin{array}{l}0.15 \\
7\end{array}$ & $\begin{array}{l}0.0 \\
78\end{array}$ & $\begin{array}{l}- \\
0.1 \\
97\end{array}$ & $\begin{array}{l}0 . \\
0 \\
0 \\
4\end{array}$ & $\begin{array}{l}0.08 \\
4\end{array}$ & $\begin{array}{l}0.92 \\
844\end{array}$ & & \\
\hline $\begin{array}{l}\text { Quie } \\
\text { scen } \\
\text { ce } \\
\text { Silen } \\
\text { ce }\end{array}$ & $\begin{array}{l}0.39 \\
9\end{array}$ & $\begin{array}{l}0.46 \\
3\end{array}$ & $\begin{array}{l}0.5 \\
09\end{array}$ & $\begin{array}{l}- \\
0.1 \\
42 \\
\end{array}$ & $\begin{array}{l}0 \\
5 \\
3 \\
\end{array}$ & $\begin{array}{l}0.12 \\
3\end{array}$ & $\begin{array}{l}0.57 \\
9\end{array}$ & $\begin{array}{l}0.92 \\
249 \\
7\end{array}$ & & $\begin{array}{l}0.09 \\
9 \\
\end{array}$ & $\begin{array}{l}0.22 \\
4\end{array}$ & $\begin{array}{l}0.4 \\
4\end{array}$ & $\begin{array}{l}0.0 \\
98\end{array}$ & $\begin{array}{l}0 . \\
1 \\
7\end{array}$ & $\begin{array}{l}0.06 \\
4\end{array}$ & $\begin{array}{l}- \\
0.19 \\
6\end{array}$ & $\begin{array}{l}0.91 \\
323 \\
6\end{array}$ & \\
\hline $\begin{array}{l}\text { Quie } \\
\text { scen } \\
\text { ce }\end{array}$ & & & & & 2 & - & & & 0.86 & & & & & $\begin{array}{l}0 . \\
0\end{array}$ & & & & $\begin{array}{l}0.86 \\
883\end{array}$ \\
\hline $\begin{array}{l}\text { Voic } \\
\mathrm{e}\end{array}$ & $\begin{array}{l}0.26 \\
5\end{array}$ & $\begin{array}{l}0.57 \\
7\end{array}$ & $\begin{array}{l}0.3 \\
01\end{array}$ & $\begin{array}{l}0.2 \\
06\end{array}$ & $\begin{array}{l}8 \\
8\end{array}$ & $\begin{array}{l}0.11 \\
6\end{array}$ & 0.26 & $\begin{array}{l}0.34 \\
2\end{array}$ & $\begin{array}{l}255 \\
4\end{array}$ & $\begin{array}{l}0.26 \\
2\end{array}$ & $\begin{array}{l}0.29 \\
5\end{array}$ & $\begin{array}{l}0.2 \\
82\end{array}$ & $\begin{array}{l}0.3 \\
46\end{array}$ & $\begin{array}{l}3 \\
5\end{array}$ & $\begin{array}{l}0.30 \\
5\end{array}$ & $\begin{array}{l}0.09 \\
4\end{array}$ & $\begin{array}{l}0.10 \\
3\end{array}$ & $\begin{array}{l}833 \\
2\end{array}$ \\
\hline
\end{tabular}

Table 3: Discriminant Validity (HTMT.90 Criterion) Ringle and Sarstedt (2015)

\begin{tabular}{|c|c|c|c|c|c|c|c|c|c|c|c|c|c|c|c|c|c|c|}
\hline \multicolumn{19}{|c|}{ Discriminant Validity (HTMT.90 Criterion) Ringle and Sarstedt (2015) } \\
\hline \multicolumn{10}{|c|}{ Gender Similarity } & \multicolumn{9}{|c|}{ Gender Dissimilarity } \\
\hline & Acq & Acq & A & $\mathrm{Be}$ & & Opp & Pro & & Qui & Acq & Acq & A & $\mathrm{Be}$ & & Opp & Pro & & Qui \\
\hline & uies & uies & ut & ne & $\mathrm{M}$ & ortu & - & Qui & esc & uies & uies & ut & ne & $\mathrm{M}$ & ortu & - & Qui & esc \\
\hline & cenc & cen & ho & vo & o & nisti & Soc & esce & enc & cenc & cen & ho & vo & o & nisti & Soc & esce & enc \\
\hline Con & $\mathrm{e}$ & $\mathrm{ce}$ & rit & le & $\mathrm{r}$ & $\mathrm{c}$ & ial & nce & $\mathrm{e}$ & $\mathrm{e}$ & ce & rit & le & $\mathrm{r}$ & $\mathrm{c}$ & ial & nce & $\mathrm{e}$ \\
\hline stru & Sile & Voi & ari & $\mathrm{nc}$ & $\mathrm{a}$ & Sile & Sile & Sile & Voi & Sile & Voi & ari & $\mathrm{nc}$ & $\mathrm{a}$ & Sile & Sile & Sile & Voi \\
\hline cts & nce & $\mathrm{ce}$ & an & $\mathrm{e}$ & 1 & nce & nce & nce & ce & nce & ce & an & $\mathrm{e}$ & 1 & nce & nce & nce & ce \\
\hline \multirow{2}{*}{\multicolumn{19}{|c|}{$\begin{array}{l}\text { Acq } \\
\text { uies }\end{array}$}} \\
\hline & & & & & & & & & & & & & & & & & & \\
\hline \multicolumn{19}{|l|}{ cenc } \\
\hline \multicolumn{19}{|l|}{$\mathrm{e}$} \\
\hline \multicolumn{19}{|l|}{ Sile } \\
\hline \multicolumn{19}{|l|}{ nce } \\
\hline \multirow{2}{*}{\multicolumn{19}{|c|}{$\begin{array}{l}\text { Acq } \\
\text { uies }\end{array}$}} \\
\hline & & & & & & & & & & & & & & & & & & \\
\hline \multicolumn{19}{|l|}{ cenc } \\
\hline e & & & & & & & & & & & & & & & & & & \\
\hline Voi & 0.43 & & & & & & & & & 0.45 & & & & & & & & \\
\hline ce & 6 & & & & & & & & & 8 & & & & & & & & \\
\hline Aut & 0.31 & 0.52 & & & & & & & & 0.18 & 0.58 & & & & & & & \\
\hline horit & 2 & 3 & & & & & & & & 9 & 2 & & & & & & & \\
\hline
\end{tabular}




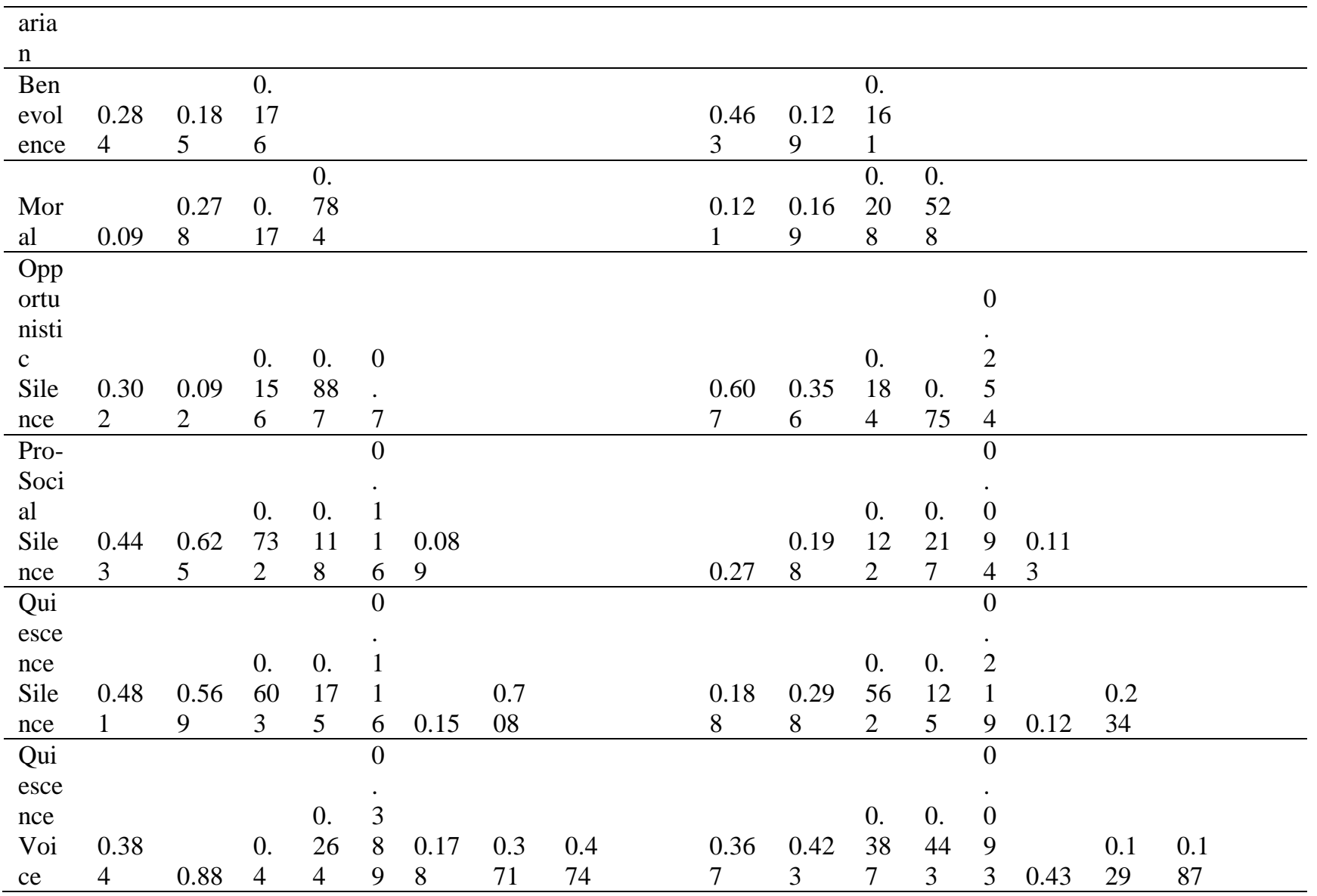

\section{Assessment of the Structural Model}

After conducting measurement analysis, structural models for gender similarity and gender dissimilarity dyads were assessed. The explanatory power of the model was tested by using $\mathrm{R}^{2}$ values (Hair et al. 2014) (results are presented in Table 4). In the behavioural research field, the $\mathrm{R}^{2}$ value of 0.2 is considered acceptable (Hair et al. 2014). Following the same, only the $\mathrm{R}^{2}$ values of acquiescence silence, quiescence voice for gender similarity and pro-social silence for gender dissimilarity can be considered low. Further, model fit was assessed by using SRMR recommended by Henseler et al., (2016) as an approximate model fit measure and it is required to be less than 0.08. The results revealed SRMR model fit values of 0.073 and 0.078 for gender similarity and gender dissimilarity respectively. Hence met the established criteria.

Table 4: $\mathrm{R}^{2}$ Values

\begin{tabular}{lll}
\hline Construct & $\mathbf{R}^{\mathbf{2}}$ for Gender Similarity & $\mathbf{R}^{\mathbf{2}}$ for Gender Dissimilarity \\
\hline Acquiescence Silence & 0.134 & 0.211 \\
\hline Acquiescence Voice & 0.238 & 0.242 \\
\hline Opportunistic Silence & 0.559 & 0.445 \\
\hline Pro-Social Silence & 0.356 & 0.057 \\
\hline Quiescence Silence & 0.267 & 0.227 \\
\hline Quiescence Voice & 0.187 & 0.264 \\
\hline
\end{tabular}

Table 5 along with figures 2 and 3 presents the assessment results of both structural models and hypothesis tests. Results are based upon 5,000 bootstraps resamples and 5,000 permutations (Henseler et al., 2016; Rasoolimanesh et al., 2017; Sarstedt et al., 2011). The results reveal that authoritarian had a positive significant effect on acquiescence silence, prosocial silence, quiescence silence, and acquiescence voice, quiescence voice of group having gender similarity. Further showed a positive significant effect of authoritarian dimension on acquiescence silence, acquiescence voice, opportunistic silence, quiescence silence, quiescence silence, and quiescence voice for gender dissimilar groups. Additionally, the results also alluded to a significant and negative effect of benevolence on the acquiescence silence and opportunistic silence for gender similar groups. Also, the negative significant effect of benevolence on acquiescence silence, opportunistic silence, pro-social silence, quiescence silence, and quiescence voice for gender dissimilar groups. Further, morality had a significant positive effect on quiescence voice for both genders in similar and dissimilar groups. 


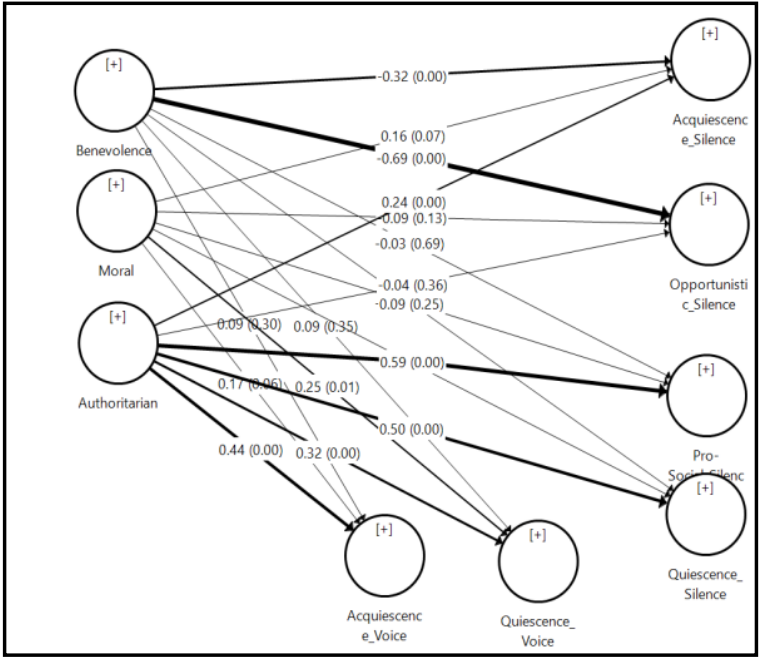

Figure 2: For Gender Similar Dyads

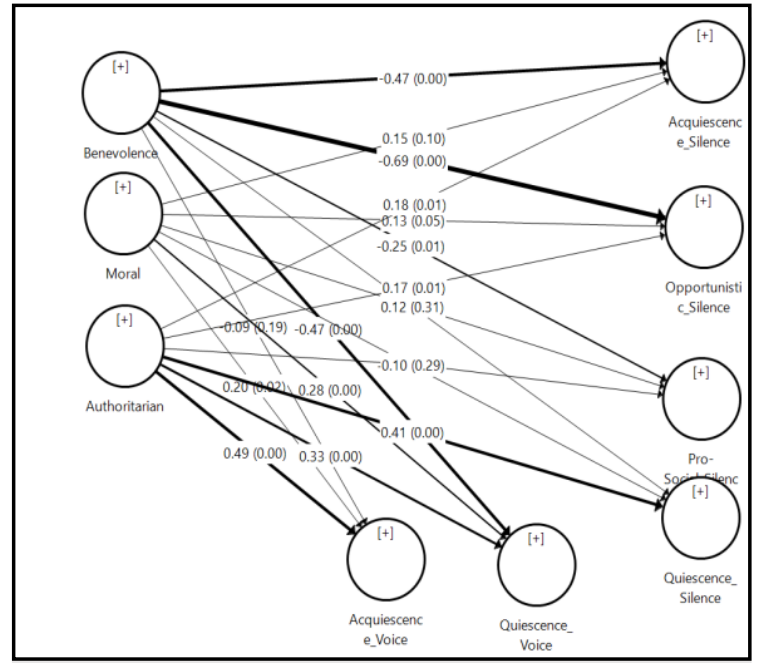

Figure 3: For Gender Dissimilar Dyads

\section{Multigroup Analysis (MGA)}

Measurement invariance of composites (MICOM) for both models was performed as a precondition for conducting MGA (Hair et al. 2014; Henseler, Ringle, and Sarstedt 2016). MICOM is a three-step process including, assessing configural invariance, ensuring compositional invariance, and assessing equality of means and variances (Henseler et al., 2016). Results revealed the partial measurement invariance of both groups (results are presented in Table 5), which is a prerequisite for comparing and interpreting the MGA (Henseler et al., 2016). But failed to establish full measurement invariance, so we were unable to pool the data for analysing basic hypotheses from $\mathrm{H} 1$ - $\mathrm{H} 3$. But the establishment of the partial measurement invariance of both groups is sufficient for analysis of similarity attraction account (Henseler et al. 2016).

Table 5: Results of Invariance Measurement Testing Using Permutation

\begin{tabular}{|c|c|c|c|c|c|c|c|c|c|c|c|}
\hline \multicolumn{12}{|c|}{ Results of Invariance Measurement Testing Using Permutation. } \\
\hline & & \multicolumn{2}{|c|}{$\begin{array}{l}\text { Composition } \\
\text { al Invariance } \\
\text { (Correlation } \\
=1 \text { ) }\end{array}$} & & \multicolumn{3}{|c|}{ Equal Mean Assessment } & \multicolumn{3}{|c|}{$\begin{array}{l}\text { Equal } \\
\text { Assessment }\end{array}$} & \\
\hline Constructs & $\begin{array}{l}\text { Config } \\
\text { ural } \\
\text { Invaria } \\
\text { nce } \\
\text { (Same } \\
\text { Algorit } \\
\text { hms } \\
\text { for } \\
\text { Both } \\
\text { Group } \\
\text { s) }\end{array}$ & $\begin{array}{l}\mathrm{C}= \\
1\end{array}$ & $\begin{array}{l}\text { Confid } \\
\text { ence } \\
\text { Interva } \\
1\end{array}$ & $\begin{array}{l}\text { Partial } \\
\text { Measure } \\
\text { ment } \\
\text { Invarian } \\
\text { ce } \\
\text { Establis } \\
\text { hed }\end{array}$ & $\begin{array}{l}\text { Differe } \\
\text { nces }\end{array}$ & $\begin{array}{l}\text { Confide } \\
\text { nce } \\
\text { Interval }\end{array}$ & $\begin{array}{l}\mathrm{Eq} \\
\text { ual }\end{array}$ & $\begin{array}{l}\text { Differe } \\
\text { nces }\end{array}$ & $\begin{array}{l}\text { Confide } \\
\text { nce } \\
\text { Interval }\end{array}$ & $\begin{array}{l}\mathrm{Eq} \\
\text { ual }\end{array}$ & $\begin{array}{l}\text { Full } \\
\text { Measure } \\
\text { ment } \\
\text { Invarian } \\
\text { ce } \\
\text { Establis } \\
\text { hed }\end{array}$ \\
\hline $\begin{array}{l}\text { Acquiescence } \\
\text { _Silence }\end{array}$ & Yes & $\begin{array}{l}0.9 \\
99\end{array}$ & $\begin{array}{l}{[0.993,} \\
1]\end{array}$ & Yes & -0.034 & $\begin{array}{l}{[-} \\
0.208,0 . \\
21]\end{array}$ & $\begin{array}{l}\mathrm{Ye} \\
\mathrm{s}\end{array}$ & 0.003 & $\begin{array}{l}{[-} \\
0.242,0 . \\
261]\end{array}$ & $\begin{array}{l}\mathrm{Ye} \\
\mathrm{s}\end{array}$ & Yes \\
\hline $\begin{array}{l}\text { Acquiescence } \\
\text { _Voice }\end{array}$ & Yes & $\begin{array}{l}1.0 \\
00\end{array}$ & $\begin{array}{l}{[0.991,} \\
1]\end{array}$ & Yes & -0.152 & $\begin{array}{l}{[-} \\
0.209,0 . \\
207]\end{array}$ & $\begin{array}{l}\mathrm{Ye} \\
\mathrm{s}\end{array}$ & 0.005 & $\begin{array}{l}{[-} \\
0.259,27 \\
1]\end{array}$ & $\begin{array}{l}\mathrm{Ye} \\
\mathrm{s}\end{array}$ & Yes \\
\hline Authoritarian & Yes & $\begin{array}{l}0.9 \\
95\end{array}$ & $\begin{array}{l}{[0.994,} \\
1]\end{array}$ & Yes & -0.271 & $\begin{array}{l}{[-} \\
0.210,0 . \\
211]\end{array}$ & No & 0.004 & $\begin{array}{l}{[-} \\
0.226,0 . \\
233]\end{array}$ & $\begin{array}{l}\mathrm{Ye} \\
\mathrm{s}\end{array}$ & No \\
\hline Benevolence & Yes & $\begin{array}{l}0.9 \\
98\end{array}$ & $\begin{array}{l}{[0.998,} \\
1]\end{array}$ & Yes & -0.499 & $\begin{array}{l}{[-} \\
0.215,0 . \\
213]\end{array}$ & No & 0.001 & $\begin{array}{l}{[-} \\
0.241,0 . \\
249]\end{array}$ & $\begin{array}{l}\mathrm{Ye} \\
\mathrm{s}\end{array}$ & No \\
\hline Moral & Yes & $\begin{array}{l}0.9 \\
99\end{array}$ & $\begin{array}{l}{[0.985,} \\
1]\end{array}$ & Yes & -0.433 & $\begin{array}{l}{[-} \\
0.198,0 . \\
208]\end{array}$ & No & 0.003 & $\begin{array}{l}{[-} \\
0.258,0 . \\
275]\end{array}$ & $\begin{array}{l}\mathrm{Ye} \\
\mathrm{s}\end{array}$ & No \\
\hline
\end{tabular}


https://doi.org/10.18510/hssr.2021.93155

\begin{tabular}{|c|c|c|c|c|c|c|c|c|c|c|c|}
\hline $\begin{array}{l}\text { Opportunistic } \\
\text { _Silence }\end{array}$ & Yes & $\begin{array}{l}0.9 \\
99\end{array}$ & $\begin{array}{l}{[0.998,} \\
1]\end{array}$ & Yes & 0.378 & $\begin{array}{l}- \\
0.213,0 . \\
209] \\
\end{array}$ & No & 0.006 & $\begin{array}{l}{[-} \\
0.23,0.2 \\
56] \\
\end{array}$ & $\begin{array}{l}\text { Ye } \\
\mathrm{s}\end{array}$ & No \\
\hline $\begin{array}{l}\text { Pro- } \\
\text { Social_Silence }\end{array}$ & Yes & $\begin{array}{l}0.9 \\
96\end{array}$ & $\begin{array}{l}{[0.993,} \\
1]\end{array}$ & Yes & $-\mathbf{- 0 . 1 5 5}$ & $\begin{array}{l}{[-} \\
0.212,0 . \\
202]\end{array}$ & $\begin{array}{l}\text { Ye } \\
\mathrm{s}\end{array}$ & 0.005 & $\begin{array}{l}{[-} \\
0.201,0 . \\
218]\end{array}$ & $\begin{array}{l}\text { Ye } \\
\mathrm{s}\end{array}$ & Yes \\
\hline $\begin{array}{l}\text { Quiescence_S } \\
\text { ilence }\end{array}$ & Yes & $\begin{array}{l}0.9 \\
95\end{array}$ & $\begin{array}{l}{[0.994,} \\
1]\end{array}$ & Yes & -0.126 & $\begin{array}{l}{[-} \\
0.212,0 . \\
212]\end{array}$ & $\begin{array}{l}\text { Ye } \\
\mathrm{s}\end{array}$ & 0.003 & $\begin{array}{l}{[-} \\
0.221,0 . \\
225]\end{array}$ & No & No \\
\hline $\begin{array}{l}\text { Quiescence_V } \\
\text { oice }\end{array}$ & Yes & $\begin{array}{l}0.9 \\
75\end{array}$ & $\begin{array}{l}{[0.955,} \\
1]\end{array}$ & Yes & 0.118 & $\begin{array}{l}- \\
0.212,0 . \\
212]\end{array}$ & $\begin{array}{l}\text { Ye } \\
\mathrm{s}\end{array}$ & 0.001 & $\begin{array}{l}- \\
0.240,0 . \\
238]\end{array}$ & $\begin{array}{l}\text { Ye } \\
\text { s }\end{array}$ & No \\
\hline
\end{tabular}

After performing MICOM, MGA was conducted by following both nonparametric methods including Henseler's MGA (Henseler et al., 2009) and the permutation test (Chin \& Dibbern, 2010). To increase the credibility of results, the study had used both recommended methods of MGA to confirm the significance/no significance of the results for identifying differences among gender similar and dissimilar dyads. Results of MGA and structural model assessment are presented in Table 6.

Table 6: Result of Structural Model and MGA Analysis

\begin{tabular}{|c|c|c|c|c|c|c|c|c|c|c|}
\hline \multicolumn{11}{|c|}{ Result of MGA Analysis } \\
\hline \multirow[b]{2}{*}{ Relationships } & \multicolumn{2}{|c|}{$\begin{array}{l}\text { Path } \\
\text { Coefficient }\end{array}$} & \multicolumn{2}{|c|}{$\begin{array}{l}\text { Confidence } \\
\text { Interval }(95 \%) \\
\end{array}$} & \multirow[b]{2}{*}{$\begin{array}{l}\text { Path } \\
\text { Coeff } \\
\text { icient } \\
\text { Differ } \\
\text { ence }\end{array}$} & \multicolumn{5}{|c|}{ P-Value Difference (One-Tailed) } \\
\hline & $\begin{array}{l}\text { Gend } \\
\text { er } \\
\text { Simil } \\
\text { arity }\end{array}$ & $\begin{array}{l}\text { Gende } \\
\mathbf{r} \\
\text { Dissim } \\
\text { ilarity }\end{array}$ & $\begin{array}{l}\text { Gender } \\
\text { Similar } \\
\text { ity }\end{array}$ & $\begin{array}{l}\text { Gender } \\
\text { Dissimi } \\
\text { larity }\end{array}$ & & $\begin{array}{l}\text { Hens } \\
\text { eler's } \\
\text { MGA }\end{array}$ & $\begin{array}{l}\text { p- } \\
\text { Value } \\
\text { Hens } \\
\text { eler's } \\
\text { MGA }\end{array}$ & $\begin{array}{l}\text { Permu } \\
\text { tation } \\
\text { Test }\end{array}$ & $\begin{array}{l}\text { Permu } \\
\text { tation } \\
\text { p- } \\
\text { Values }\end{array}$ & $\begin{array}{l}\text { Supp } \\
\text { orted }\end{array}$ \\
\hline $\begin{array}{l}\text { Authoritarian -> } \\
\text { Acquiescence_Silence }\end{array}$ & $\begin{array}{l}0.236 \\
* *\end{array}$ & $0.178^{*}$ & $\begin{array}{l}{[0.096,0} \\
.357]\end{array}$ & $\begin{array}{l}{[0.357,0} \\
.026]\end{array}$ & 0.058 & 0.058 & 0.280 & 0.058 & 0.557 & $\begin{array}{l}\mathrm{No} / \mathrm{N} \\
\mathrm{o}\end{array}$ \\
\hline $\begin{array}{l}\text { Authoritarian -> } \\
\text { Acquiescence_Voice }\end{array}$ & $\begin{array}{l}0.444 \\
* *\end{array}$ & $\begin{array}{l}0.491 * \\
*\end{array}$ & $\begin{array}{l}{[0.313, .} \\
545]\end{array}$ & $\begin{array}{l}{[0.545,0} \\
.357]\end{array}$ & -0.047 & 0.047 & 0.722 & -0.047 & 0.573 & $\begin{array}{l}\mathrm{No} / \mathrm{N} \\
\mathrm{o}\end{array}$ \\
\hline $\begin{array}{l}\text { Authoritarian } \quad-> \\
\text { Opportunistic_Silenc } \\
\text { e }\end{array}$ & $\begin{array}{l}- \\
0.044\end{array}$ & $\begin{array}{l}0.174 * \\
*\end{array}$ & $\begin{array}{l}{[-} \\
0.144,0 . \\
044]\end{array}$ & $\begin{array}{l}{[0.044,0} \\
.049]\end{array}$ & -0.219 & 0.219 & 0.997 & -0.219 & 0.004 & $\begin{array}{l}\mathrm{No} / \mathrm{N} \\
\mathrm{o}\end{array}$ \\
\hline $\begin{array}{l}\text { Authoritarian -> Pro- } \\
\text { Social Silence }\end{array}$ & $\begin{array}{l}0.593 \\
* * \\
\end{array}$ & 0.099 & $\begin{array}{l}{[0.481,0} \\
.677]\end{array}$ & $\begin{array}{l}{[0.677,-} \\
0.105]\end{array}$ & 0.494 & 0.494 & 0.000 & 0.494 & 0.000 & $\begin{array}{l}\text { Yes/ } \\
\text { Yes }\end{array}$ \\
\hline $\begin{array}{ll}\text { Authoritarian } & \text {-> } \\
\text { Quiescence Silence }\end{array}$ & $\begin{array}{l}0.498 \\
* *\end{array}$ & $\begin{array}{l}0.411^{*} \\
*\end{array}$ & $\begin{array}{l}{[0.382,0} \\
.593]\end{array}$ & $\begin{array}{l}{[0.593,0} \\
.255]\end{array}$ & 0.087 & 0.087 & 0.162 & 0.087 & 0.310 & $\begin{array}{l}\mathrm{No} / \mathrm{N} \\
\mathrm{o}\end{array}$ \\
\hline $\begin{array}{ll}\text { Authoritarian } & -> \\
\text { Quiescence Voice }\end{array}$ & $\begin{array}{l}0.323 \\
* *\end{array}$ & $\begin{array}{l}0.329 * \\
*\end{array}$ & $\begin{array}{l}{[0.186,0} \\
.431]\end{array}$ & $\begin{array}{l}{[0.432,0} \\
.177]\end{array}$ & -0.006 & 0.006 & 0.528 & -0.006 & 0.953 & $\begin{array}{l}\mathrm{No} / \mathrm{N} \\
\mathrm{o}\end{array}$ \\
\hline $\begin{array}{l}\text { Benevolence -> } \\
\text { acquiescence Silence }\end{array}$ & $\begin{array}{l}- \\
0.322 \\
* *\end{array}$ & $-474 * *$ & $\begin{array}{l}{[-} \\
0.476,- \\
0.125] \\
\end{array}$ & $\begin{array}{l}{[-} \\
0.125,- \\
0.607] \\
\end{array}$ & 0.153 & 0.153 & 0.091 & 0.153 & 0.196 & $\begin{array}{l}\mathrm{No} / \mathrm{N} \\
\mathrm{o}\end{array}$ \\
\hline $\begin{array}{l}\text { Benevolence } \\
\text { acquiescence Voice }\end{array}$ & 0.095 & -0.09 & $\begin{array}{l}- \\
0.077,0 . \\
273]\end{array}$ & $\begin{array}{l}{[0.273,-} \\
0.229]\end{array}$ & 0.185 & 0.185 & 0.051 & 0.185 & 0.107 & $\begin{array}{l}\mathrm{No} / \mathrm{N} \\
\mathrm{o}\end{array}$ \\
\hline $\begin{array}{l}\text { Benevolence } \quad-> \\
\text { Opportunistic Silence }\end{array}$ & $\begin{array}{l}- \\
0.692 \\
* *\end{array}$ & $\begin{array}{l}- \\
0.693 * \\
* \\
\end{array}$ & $\begin{array}{l}{[-} \\
0.789,- \\
0.564] \\
\end{array}$ & $\begin{array}{l}{[-} \\
0.565,- \\
0.792]\end{array}$ & 0.001 & 0.001 & 0.492 & 0.001 & 0.987 & $\begin{array}{l}\mathrm{No} / \mathrm{N} \\
\mathrm{o}\end{array}$ \\
\hline $\begin{array}{l}\text { Benevolence -> Pro- } \\
\text { Social_Silence }\end{array}$ & - & $\begin{array}{l}- \\
0.249 * \\
*\end{array}$ & $\begin{array}{l}- \\
0.179,0 . \\
127] \\
\end{array}$ & $\begin{array}{l}{[0.127,-} \\
0.408]\end{array}$ & 0.218 & 0.218 & 0.039 & 0.218 & 0.108 & $\begin{array}{l}\mathrm{No} / \mathrm{N} \\
\mathrm{o}\end{array}$ \\
\hline $\begin{array}{l}\text { Benevolence -> } \\
\text { Quiescence Silence }\end{array}$ & - & 0.168 & $\begin{array}{l}- \\
0.294,0 . \\
074] \\
\end{array}$ & $\begin{array}{l}{[0.074,-} \\
0.014]\end{array}$ & -0.280 & 0.280 & 0.985 & -0.280 & 0.030 & $\begin{array}{l}\text { Yes/ } \\
\text { Yes }\end{array}$ \\
\hline $\begin{array}{l}\text { Benevolence } \\
\text { Quiescence Voice }\end{array}$ & 0.088 & $\begin{array}{l}- \\
0.465 * \\
*\end{array}$ & $\begin{array}{l}{[-} \\
0.091,0 . \\
265]\end{array}$ & $\begin{array}{l}{[0.265,-} \\
0.601]\end{array}$ & 0.554 & 0.554 & 0.000 & 0.554 & 0.000 & $\begin{array}{l}\text { Yes/ } \\
\text { Yes }\end{array}$ \\
\hline Moral & 0.162 & 0.145 & {$[-$} & {$[0.331,-$} & 0.017 & 0.017 & 0.446 & 0.017 & 0.894 & $\mathrm{No} / \mathrm{N}$ \\
\hline
\end{tabular}




\begin{tabular}{|c|c|c|c|c|c|c|c|c|c|c|}
\hline Acquiescence Silence & & & $\begin{array}{l}0.026,0 . \\
331]\end{array}$ & $0.037]$ & & & & & & o \\
\hline $\begin{array}{l}\text { Moral } \\
\text { Acquiescence Voice }\end{array}$ & 0.171 & 0.198 & $\begin{array}{l}{[-} \\
0.014,0 . \\
332]\end{array}$ & $\begin{array}{l}{[0.332,0} \\
.031]\end{array}$ & -0.027 & 0.027 & 0.587 & -0.027 & 0.818 & $\begin{array}{l}\mathrm{No} / \mathrm{N} \\
\mathrm{o}\end{array}$ \\
\hline $\begin{array}{l}\text { Moral -> } \\
\text { Opportunistic Silence }\end{array}$ & - & 0.127 & $\begin{array}{l}{[-} \\
0.210,0 . \\
018]\end{array}$ & $\begin{array}{l}{[0.018,0} \\
.004]\end{array}$ & -0.218 & 0.218 & 0.994 & -0.218 & 0.016 & $\begin{array}{l}\text { Yes/ } \\
\text { Yes }\end{array}$ \\
\hline $\begin{array}{l}\text { Moral -> Pro-Social } \\
\text { Silence }\end{array}$ & 0.091 & 0.123 & $\begin{array}{l}{[-} \\
0.062,0 . \\
236]\end{array}$ & $\begin{array}{l}{[0.236,-} \\
0.176]\end{array}$ & -0.032 & 0.032 & 0.609 & -0.032 & 0.808 & $\begin{array}{l}\mathrm{No} / \mathrm{N} \\
\mathrm{o}\end{array}$ \\
\hline $\begin{array}{l}\text { Moral -> Quiescence } \\
\text { Silence }\end{array}$ & 0.041 & -0.175 & $\begin{array}{l}{[-} \\
0.144,0 . \\
221]\end{array}$ & $\begin{array}{l}{[0.221,-} \\
0.316]\end{array}$ & 0.217 & 0.217 & 0.043 & 0.217 & 0.096 & $\begin{array}{l}\mathrm{No} / \mathrm{N} \\
\mathrm{o}\end{array}$ \\
\hline $\begin{array}{l}\text { Moral -> Quiescence } \\
\text { Voice }\end{array}$ & $\begin{array}{l}0.25^{*} \\
* \\
\end{array}$ & $0.28 * *$ & $\begin{array}{l}{[0.042,0} \\
.404]\end{array}$ & $\begin{array}{l}{[0.404,0} \\
.116]\end{array}$ & -0.030 & 0.030 & 0.595 & -0.030 & 0.803 & $\begin{array}{l}\mathrm{No} / \mathrm{N} \\
\mathrm{o}\end{array}$ \\
\hline
\end{tabular}

Results of both methods shown the significant differences in the effects of authoritarian on pro-social silence, benevolence on quiescence silence, benevolence on quiescence voice, and morality on opportunistic silence for both groups. Further, results alluded to the nonsignificant differences between other path coefficients and relationships.

\section{DISCUSSION \& CONCLUSION}

The researchers attempted to analyse and compare the effect of paternalism leadership dimensions on employee voice and silence behaviour along with gender similar and dissimilar dyadic relationships among supervisor and subordinate. Results revealed that authoritarian behaviour of leader fosters silence behaviour both in gender similar/dissimilar dyadic. The current study also supports that Authoritarian leadership induces quiescent silence among subordinates, supported by Guo et al., (2018) for both genders similar and dissimilar dyads. Morsch et al., (2020), also reported a significant positive relationship between negative abusive supervision and employee quiescent silence behaviour. Additionally, authoritative behaviour encourages opportunistic silence among subordinates in gender dissimilar dyads. That is a nonproductive behaviour among university staff. This might limit knowledge sharing and the development of new knowledge. Further, results showed that authoritative leader behaviour fosters acquiescence voice that is an ignorant behaviour, and quiescence voice that is a defensive behaviour in gender similar groups. Chen, (2017), also found a positive but insignificant relationship between authoritative leadership and acquiescence voice and quiescence voice.

Moreover, the benevolence of the leader showed a negative significant effect on acquiescent silence among both groups. It reveals that a leader's benevolence toward subordinates will promote individual interest in the current situation and employees might encourage to participate and provoke themselves to found new ways. Further, benevolence also showed a significant negative relation with opportunistic silence implying that leader's benevolence toward subordinates encourages them to share their knowledge and use their expertise to sort out problems and bring new opportunities. Furthermore, the benevolence of a leader has also been proven to be negatively associated with prosocial silence and quiescent voice for gender dissimilar groups. It indicates that leaders' perceived benevolence discourages employees to opt for defensive behaviour that is also supported by Chen (2017). This perceived benevolence may also encourage employees to discuss matters rather than keeping silent for short-term benefits as they expect this will not harm their colleague rather supervisor will handle the issue with personal care. Additionally, the morality of the leader revealed a positive significant effect on the quiescent voice. A quiescent voice is backed by the defensive motive of the employee. Here context and issues need further investigation as it might be possible that employees expect the fair investigation of sensitive issues and might try to raise voices to protect or to justify their own perspective.

The main contribution of this study is conducting MGA for gender similar dyads and dissimilar dyads. The results revealed significant differences across both groups for the effect of authoritarian on pro-social silence, benevolence on quiescence silence and quiescence voice, and morality on opportunistic silence. Although the relationships were not strongly identified but the results gave a glimpse about the direction of relationships. The strong positive effect of authoritative leadership behaviour on pro-social silence for gender similar groups turned into the insignificant relationship for gender dissimilar group revealing the power of similarity attraction account. Similarly, the weaker positive effect of benevolence on defensive voice for gender similar groups turned into a significantly negative relationship for gender dissimilar groups. It revealed the role of supervisor's benevolence on employee's defensive behaviour that might produce positive effects beyond similarity attraction boundaries

\section{THEORETICAL AND PRACTICAL IMPLICATIONS}

The results of the study contribute both practically and theoretically. From the practical side, it will help leaders and policymakers to understand the effect of culturally embedded and least researched leaders' behaviour on employee voice and silence behaviour especially in the presence of gender diversity. 
Further, the context of the study is universities that are known to generate knowledge and inputs to policymakers. So, developing an understanding of the interactions of culturally embedded leadership style, gender diversity, and employee voice/silence behaviour is vital to investigate and understand for better policy development. Further, leaders of higher educational institutions need to understand how their behaviour impacts subordinate's decision to speak or remain silent at the workplace.

On a theoretical level, it is the contribution of the study to investigate the role of similarity attraction account among the interaction of leader behaviour and employee voice/silence behaviour. As it is among the pioneering study to investigate the impact of similarity attraction account on employee voice or silence behaviour in the presence of culturally embedded leadership style.

\section{LIMITATIONS AND RECOMMENDATIONS}

The study also has some limitations. First, the study used a cross-sectional design to collect data, which limits the researcher's ability to claim causal effects. Future studies can work on longitudinal and experimental designs to test causal relationships. Second, the study relied on self-response from employees, other researchers can collect data from supervisor and subordinates to reduce response biasness.

Third, this study was conducted in public sector universities of Balochistan, which limited the generalizability of results to other university settings or other types of organizations. To enhance the generalizability other researcher can replicate the study in other sector to validate results. Fourth, there can be many other variables that can interact with the paternalistic dimension to determine voice or silence behaviour, such as the organization's structure, climate, and/or culture voice mechanisms and nature of the issue, etc. that can be considered for further enhancing the concepts.

\section{AUTHORS' CONTRIBUTION}

Saadia Babur is the main contributor who developed the main theme related to the concept under consideration. Zainab Bibi performed the drafting and revising of the entire manuscript. Jahanvash Karim performed the statistical procedures and interpretation

\section{REFERENCE}

1. Abrams, D., Wetherell, M., Cochrane, S., Hogg, M. A., \& Turner, J. C. (1990). Knowing what to think by knowing who you are: Self-categorization and the nature of norm formation, conformity and group polarization. British Journal of Social Psychology, 29(2), 97-119. https://doi.org/10.1111/j.2044-8309.1990.tb00892.x

2. Arif, S., \& Ilyas, M. (2013). Quality of work-life model for teachers of private universities in Pakistan. Quality Assurance in Education, 21(3), 282-298. https://doi.org/10.1108/QAE-Feb-2012-0006

3. Aycan, Z., Kanungo, R., Mendonca, M., Yu, K., Deller, J., Stahl, G., \& Kurshid, A. (2000). Impact of Culture on Human Resource Management Practices: A 10-Country Comparison. Applied Psychology, 49(1), $192-221$. https://doi.org/10.1111/1464-0597.00010

4. Aycan, Z., Kanungo, R. N., \& Sinha, J. B. P. (1999). Organizational culture and human resource management practices: The model of culture fit. Journal of Cross-Cultural Psychology, 30(4), 501-526. https://doi.org/10 $.1177 / 0022022199030004006$

5. Bano, S., Ramzan, S., Anjum, M. A., \& Dapeng, L. (2017). Does Perceived Social Support Mediate the Relationship of Perceived Organizational Support \& Job Satisfaction? A Structural Equation Modeling Approach. Journal of Managerial Sciences, 11(3), 105-118.

6. Bibi, Z., Karim, J., \& Din, S. U. (2013). Workplace Incivility and Counterproductive Work Behavior: Moderating Role of Emotional Intelligence. Pakistan Journal of Psychological Research, 28(2), 317-334.

7. Brinsfield, C. T. (2013). Employee Silence Motives: Investigation of Dimensionality and Development of Measures: Employee Silence Motives. Journal of Organizational Behavior, 34(5), 671-697. https://doi. org/10.1002/job.1829

8. Brown, M. E., Treviño, L. K., \& Harrison, D. A. (2005). Ethical leadership: A social learning perspective for construct development and testing. Organizational Behavior and Human Decision Processes, 97(2), 117-134. https://doi.org/10.1016/j.obhdp.2005.03.002

9. Campbell, E. G., Clarridge, B. R., Gokhale, M., Birenbaum, L., Hilgartner, S., Holtzman, N. A., \& Blumenthal, D. (2002). Data Withholding in Academic Genetics: Evidence From a National Survey. JAMA, $287(4), 473$. https://doi.org/10.1001/jama.287.4.473

10. Chan, S. C. (2014). Paternalistic leadership and employee voice: Does information sharing matter? Human Relations, 67(6), 667-693. https://doi.org/10.1177/0018726713503022

11. Chan, S. C. H., Huang, X., Snape, E., \& Lam, C. K. (2013). The Janus face of paternalistic leaders: Authoritarianism, benevolence, subordinates' organization-based self-esteem, and performance. Journal of Organizational Behavior, 34(1), 108-128. https://doi.org/10.1002/job.1797

12. Chen, A. S.-Y., \& Hou, Y.-H. (2016). The effects of ethical leadership, voice behavior and climates for innovation on creativity: A moderated mediation examination. The Leadership Quarterly, 27(1), 1-13. https://doi.org/10.1016/j.leaqua.2015.10.007

13. Chen, S.-C. (2017). Paternalistic leadership and cabin crews' upward safety communication: The motivation of 
voice behavior. Journal of Air Transport Management, 62, 44-53. https://doi.org/10.1016/j.jairtrama n.2017.02.007

14. Cheng, B.-S., Chou, L.-F., Wu, T.-Y., Huang, M.-P., \& Farh, J.-L. (2004). Paternalistic leadership and subordinate responses: Establishing a leadership model in Chinese organizations: Paternalistic leadership. Asian Journal of Social Psychology, 7(1), 89-117. https://doi.org/10.1111/j.1467-839X.2004.00137.x

15. Chin, W. W. (2010). How to Write Up and Report PLS Analyses. In V. Esposito Vinzi, W. W. Chin, J. Henseler, \& H. Wang (Eds.), Handbook of Partial Least Squares (pp. 655-690). Springer Berlin Heidelberg. https://doi.org/10.1007/978-3-540-32827-8_29

16. Chin, W. W., \& Dibbern, J. (2010). An Introduction to a Permutation Based Procedure for Multi-Group PLS Analysis: Results of Tests of Differences on Simulated Data and a Cross Cultural Analysis of the Sourcing of Information System Services Between Germany and the USA. In V. Esposito Vinzi, W. W. Chin, J. Henseler, \& H. Wang (Eds.), Handbook of Partial Least Squares (pp. 171-193).

17. Chuan, C. L. (2006). Sample Size Estimation Using Krejcie and Morgan and Cohen Statistical Power Analysis: A Comparison. Jurnal Penyelidikan IPBL, 7(1), 9.

18. Cohen, J. (1992). Quantitative Methods in Psychology. Psychological Bulletin, 112(1), 5.

19. Detert, J. R., \& Burris, E. R. (2007). Leadership Behavior and Employee Voice: Is the Door Really Open? Academy of Management Journal, 50(4), 869-884. https://doi.org/10.5465/amj.2007.26279183

20. Duffy, M. K., \& Ferrier, W. J. (2003). Birds of a Feather...?: How Supervisor-Subordinate Dissimilarity Moderates the Influence of Supervisor Behaviors on Workplace Attitudes. Group \& Organization Management, 28(2), 217-248. https://doi.org/10.1177/1059601103028002003

21. Dutton, J. E., Ashford, S. J., \& Lawrenge, K. A. (2001). Moves That Matter: Issue Selling and Organizational Change. Academy of Management Journal, 44(4), 716-736.

22. Dyne, L. V., Ang, S., \& Botero, I. C. (2003). Conceptualizing Employee Silence and Employee Voice as Multidimensional Constructs. Journal of Management Studies, 40(6), 1359-1392. https://doi.org/10.1111/14676486.00384

23. Edwards, J. R., Peterson, K. D., Mu, Y., Banerjee, S., Allen-Bridson, K., Morrell, G., Dudeck, M. A., Pollock, D. A., \& Horan, T. C. (2009). National Healthcare Safety Network (NHSN) report: Data summary for 2006 through 2008, issued December 2009. American Journal of Infection Control, 37(10), 783-805. https://doi.or g/10.1016/j.ajic.2009.10.001

24. Emerson, R. (1962). Power-Dependence Relations. American Sociological Review, 27(1), 31-41.

25. Emerson, R. M. (1976). Social Exchange Theory. Annual Review of Sociology, 16 (2), 335-362.

26. Farh, J.-L., \& Cheng, B.-S. (2000). A Cultural Analysis of Paternalistic Leadership in Chinese Organizations. In J. T. Li, A. S. Tsui, \& E. Weldon (Eds.), Management and Organizations in the Chinese Context (pp. 84 127). Palgrave Macmillan UK. https://doi.org/10.1057/9780230511590_5

27. Farrukh, M., Lee, J. W. C., \& Shahzad, I. A. (2019). Intrapreneurial behavior in higher education institutes of Pakistan: The role of leadership styles and psychological empowerment. Journal of Applied Research in Higher Education, 11(2), 273-294. https://doi.org/10.1108/JARHE-05-2018-0084

28. Ferris, D. L., Brown, D. J., \& Heller, D. (2009). Organizational supports and organizational deviance: The mediating role of organization-based self-esteem. Organizational Behavior and Human Decision Processes, 108(2), 279-286. https://doi.org/10.1016/j.obhdp.2008.09.001

29. Gelfand, M. J., Erez, M., \& Aycan, Z. (2007). Cross-Cultural Organizational Behavior. Annual Review of Psychology, 58(1), 479-514. https://doi.org/10.1146/annurev.psych.58.110405.085559

30. Gorodnichenko, Y., \& Roland, G. (2012). Understanding the Individualism-Collectivism Cleavage and Its Effects: Lessons from Cultural Psychology. In M. Aoki, T. Kuran, \& G. Roland (Eds.), Institutions and Comparative Economic Development (pp. 213-236). Palgrave Macmillan UK. https://doi.org/10.1057/9781 $137034014 \_12$

31. Guo, L., Decoster, S., Babalola, M. T., De Schutter, L., Garba, O. A., \& Riisla, K. (2018). Authoritarian leadership and employee creativity: The moderating role of psychological capital and the mediating role of fear and defensive silence. Journal of Business Research, 92, 219-230. https://doi.org/10.1016/j.jbusr es.2018.07.034

32. Hair, J. F., Black, Jr. W. C., Babin, B. J., \& Anderson, R. E. (2014). Multivariate data analysis (7. ed., Pearson new internat. ed). Pearson.

33. Hair, J. F., Ringle, C. M., \& Sarstedt, M. (2011). PLS-SEM: Indeed a Silver Bullet. Journal of Marketing Theory and Practice, 19(2), 139-152. https://doi.org/10.2753/MTP1069-6679190202

34. He, P., Peng, Z., Zhao, H., \& Estay, C. (2019). How and When Compulsory Citizenship Behavior Leads to Employee Silence: A Moderated Mediation Model Based on Moral Disengagement and SupervisorSubordinate Guanxi Views. Journal of Business Ethics, 155(1), 259-274. https://doi.org/10.1007/s10551-0173550-2

35. Henseler, J., Ringle, C. M., \& Sarstedt, M. (2015). A new criterion for assessing discriminant validity in variance-based structural equation modeling. Journal of the Academy of Marketing Science, 43(1), 115-135. https://doi.org/10.1007/s11747-014-0403-8

36. Henseler, J., Ringle, C. M., \& Sarstedt, M. (2016). Testing measurement invariance of composites using partial 
least squares. International Marketing Review, 33(3), 405-431. https://doi.org/10.1108/IMR-09-2014-0304

37. Hernaus, T., Cerne, M., Connelly, C., Poloski Vokic, N., \& Škerlavaj, M. (2019). Evasive knowledge hiding in academia: When competitive individuals are asked to collaborate. Journal of Knowledge Management, 23(4), 597-618. https://doi.org/10.1108/JKM-11-2017-0531

38. Jackson, S. E., Brett, J. F., Sessa, V. I., Cooper, D. M., \& et al. (1991). Some differences make a difference: Individual dissimilarity and group heterogeneity as correlates of recruitment, promotions, and turnover. Journal of Applied Psychology, 76(5), 675-689. https://doi.org/10.1037/0021-9010.76.5.675

39. Judge, T. A., \& Ferris, G. R. (1993). Social Context of Performance Evaluation Decisions. The Academy of Management Journal, 36(1), 80-105. https://doi.org/10.2307/256513

40. Karim, J., Bibi, Z., \& Rehman, S. U. (2015). Emotional Intelligence and Perceived Work-related Outcomes: Mediating Role of Workplace Incivility Victimization. Pakistan Journal of Psychological Research, 30(1), 2137.

41. Kerfoot, D., \& Knights, D. (1993). management, masculinity and manipulation: from paternalism to corporate strategy in financial services in britain*. Journal of Management Studies, 30(4), 659-677. https://doi.org/1 0.1111/j.1467-6486.1993.tb00320.x

42. Kline, R. B. (2011). Principles and practice of structural equation modeling (3rd ed). Guilford Press.

43. Knoll, M., \& van Dick, R. (2013). Do I Hear the Whistle...? A First Attempt to Measure Four Forms of Employee Silence and Their Correlates. Journal of Business Ethics, 113(2), 349-362. https://doi.org/ 10.1007/s10551-012-1308-4

44. Kurzon, D. (1995). The right of silence: A socio-pragmatic model of interpretation. Journal of Pragmatics, 23(1), 55-69. https://doi.org/10.1016/0378-2166(94)00036-E

45. Lau, D. C., \& Murnighan, J. K. (2005). Interactions Within Groups and Subgroups: The Effects of Demographic Faultlines. Academy of Management Journal, 48(4), 645-659. https://doi.org/10.546 5/amj.2005.17843943

46. Liden, R. C., Wayne, S. J., \& Stilwell, D. (1993). A longitudinal study on the early development of leadermember exchanges. Journal of Applied Psychology, 78(4), 662-674. https://doi.org/10.1037/00219010.78.4.662

47. Liu, W., Zhu, R., \& Yang, Y. (2010). I warn you because I like you: Voice behavior, employee identifications, and transformational leadership. The Leadership Quarterly, 21(1), 189-202. https://doi.org/10.1016/ j.leaqua.2009.10.014

48. Lyon, D. (2002). Surveillance Studies: Understanding visibility, mobility and the phenetic fix. Surveillance \& Society, 1(1), 1-7. https://doi.org/10.24908/ss.v1i1.3390

49. Maynes, T. D., \& Podsakoff, P. M. (2014). Speaking more broadly: An examination of the nature, antecedents, and consequences of an expanded set of employee voice behaviors. Journal of Applied Psychology, 99(1), 87112. https://doi.org/10.1037/a0034284

50. Milliken, F. J., Morrison, E. W., \& Hewlin, P. F. (2003). An Exploratory Study of Employee Silence: Issues that Employees Don't Communicate Upward and Why*. Journal of Management Studies, 40(6), 1453-1476. https://doi.org/10.1111/1467-6486.00387

51. Morrison, E. W., \& Milliken, F. J. (2000). Organizational Silence: A Barrier to Change and Development in a Pluralistic World. The Academy of Management Review, 25(4), 706. https://doi.org/10.2307/259200

52. Morrison, E. W., Wheeler-Smith, S. L., \& Kamdar, D. (2011). Speaking up in groups: A cross-level study of group voice climate and voice. Journal of Applied Psychology, 96(1), 183-191. https://doi.org/10.1 $\underline{037 / \mathrm{a} 0020744}$

53. Morsch, J., Dijk, D. van, \& Kodden, B. (2020). The Impact of Perceived Psychological Contract Breach, Abusive Supervision, and Silence on Employee Well-being. Journal of Applied Business and Economics, 22(2), 37-53. https://doi.org/10.33423/jabe.v22i2.2799

54. O’Reilly, C. A., Caldwell, D. F., \& Barnett, W. P. (1989). Work Group Demography, Social Integration, and Turnover. Administrative Science Quarterly, 34(1), 21-37. https://doi.org/10.2307/2392984

55. Pfeffer, J., \& Salancik, G. R. (2003). The External Control of Organizations: A Resource Dependence Perspective. Stanford University Press.

56. Pinder, C. C., \& Harlos, K. P. (2001). Employee silence: Quiescence and acquiescence as responses to perceived injustice. In Research in Personnel and Human Resources Management (Vol. 20, pp. 331-369). Emerald (MCB UP ). https://doi.org/10.1016/S0742-7301(01)20007-3

57. Prajapati, B., Dunne, M., \& Armstrong, R. (2010). Sample size estimation and statistical power analyses. Optometry Today, 16(7), 9.

58. Rasoolimanesh, S. M., Roldán, J. L., Jaafar, M., \& Ramayah, T. (2017). Factors Influencing Residents' Perceptions toward Tourism Development: Differences across Rural and Urban World Heritage Sites. Journal of Travel Research, 56(6), 760-775. https://doi.org/10.1177/0047287516662354

59. Rigdon, E. E. (2016). Choosing PLS path modeling as analytical method in European management research: A realist perspective. European Management Journal, 34(6), 598-605. https://doi.org/10.1016/j.emj.2016.05.006

60. Sarstedt, M., Henseler, J., \& Ringle, C. M. (2011). Multigroup Analysis in Partial Least Squares (PLS) Path Modeling: Alternative Methods and Empirical Results. In M. Sarstedt, M. Schwaiger, \& C. R. Taylor (Eds.), 
Advances in International Marketing (Vol. 22, pp. 195-218). Emerald Group Publishing Limited. https://doi.org/10.1108/S1474-7979(2011)0000022012

61. Saunders, M. N. K., Lewis, P., \& Thornhill, A. (2009). Research methods for business students (5th ed). Prentice Hall.

62. Tajfel, H., \& Turner, J. F. C. (2004). The Social Identity Theory of Intergroup Behavior. https://doi.org/10. 4324/9780203505984-16

63. Tangirala, S., \& Ramanujam, R. (2008). Employee Silence on Critical Work Issues: The Cross Level Effects of Procedural Justice Climate. Personnel Psychology, 61(1), 37-68. https://doi.org/10.1111/j.17446570.2008.00105.x

64. Tsui, A. S., \& O’Reilly, C. A. (1989). Beyond Simple Demographic Effects: The Importance of Relational Demography in Superior-Subordinate Dyads. The Academy of Management Journal, 32(2), $402-423$. https://doi.org/10.2307/256368

65. van Gils, S., Van Quaquebeke, N., van Knippenberg, D., van Dijke, M., \& De Cremer, D. (2015). Ethical leadership and follower organizational deviance: The moderating role of follower moral attentiveness. The Leadership Quarterly, 26(2), 190-203. https://doi.org/10.1016/j.leaqua.2014.08.005

66. Wang, A.-C., Hsieh, H.-H., Tsai, C.-Y., \& Cheng, B.-S. (2012). Does Value Congruence Lead to Voice? Cooperative Voice and Cooperative Silence under Team and Differentiated Transformational Leadership. Management and Organization Review, 8(2), 341-370. https://doi.org/10.1111/j.1740-8784.2011.00255.x

67. Whitener, E. M., Brodt, S. E., Korsgaard, M. A., \& Werner, J. M. (1998). Managers as Initiators of Trust: An Exchange Relationship Framework for Understanding Managerial Trustworthy Behavior. https://doi.org /10.5465/amr.1998.926624

68. Wu, M., Huang, X., Li, C., \& Liu, W. (2011). Perceived Interactional Justice and Trust-in-supervisor as Mediators for Paternalistic Leadership. Management and Organization Review, 8(1), 97-121. https://doi.org 10.1111/j.1740-8784.2011.00283.x

69. Zenger, T. R., \& Lawrence, B. S. (1989). Organizational Demography: The Differential Effects of Age and Tenure Distributions on Technical Communication. Academy of Management Journal, 32(2), 353-376. https://doi.org/10.5465/256366

70. Zhang, A. Y., Tsui, A. S., \& Wang, D. X. (2011). Leadership behaviors and group creativity in Chinese organizations: The role of group processes. The Leadership Quarterly, 22(5), 851-862. https://doi.org/10 $.1016 /$ j.leaqua.2011.07.007 